\title{
NEURAL NETWORK BASED REPRESENTATION OF UH-60A PILOT AND HUB ACCELERATIONS
}

\author{
Sesi Kottapalli \\ Aeromechanics Branch \\ Army/NASA Rotorcraft Division \\ NASA Ames Research Center \\ Moffett Field, California
}

\begin{abstract}
Neural network relationships between the full-scale, experimental hub accelerations and the corresponding pilot floor vertical vibration are studied. -The present physics-based, quantitative effort represents an initial systematic study on the UH-60A Black Hawk hub accelerations. The NASA Army UH-60A Airloads Program flight test database was used. A "maneuvereffect-factor (MEF)," derived using the roll-angle and the pitch-rate, was used. Three neural network based representation-cases were considered. The pilot floor vertical vibration was considered in the first case and the hub accelerations were separately considered in the second case. The third case considered both the hub accelerations and the pilot floor vertical vibration. Neither the advance ratio nor the gross weight alone could be used to predict the pilot floor vertical vibration. However, the advance ratio and the gross weight together could be used to predict the pilot floor vertical vibration over the entire flight envelope. The hub accelerations data were modeled and found to be of very acceptable quality. The hub accelerations alone could not be used to predict the pilot floor vertical vibration. Thus, the hub accelerations alone do not drive the pilot floor vertical vibration. However, the hub accelerations, along with either the advance ratio or the gross weight or both, could be used to satisfactorily predict the pilot floor vertical vibration. The hub accelerations are clearly a factor in determining the pilot floor vertical vibration.
\end{abstract}

\section{Notation}

MEF Maneuver effect factor, Equation 1.

MIMO Multiple-input, multiple-output

MISO Multiple-input, single-output

Presented at the American Helicopter Society Aeromechanics Specialists' Meeting, Atlanta, Georgia, November 13-14, 2000. Copyright 10 by the American Helicopter Society, Inc. All rights reserved.

\author{
PVV Peak, 4P pilot floor vertical vibration, \\ g's \\ SISO Single-input, single-output

\section{Introduction}

For helicopters, the relationships between the rotor hub accelerations and the fuselage vibration are nonlinear and involve many variables. Here, fuselage vibration is defined as the $\mathrm{N} / \mathrm{rev}$ fuselage acceleration due to the main rotor, where $\mathrm{N}$ is the number of main rotor blades (presently, $\mathrm{N}=4$ ). For the UH-60A flight test data that were considered in Ref. 1, one of the conclusions was that the fuselage vibration trends qualitatively matched those of the hub accelerations. Reference 1 did not present any quantitative representations for the hub accelerations. Also, in Ref. 1, the relationships between the hub accelerations and the fuselage vibrations were not quantified.

The present study is the first systematic effort that considers hub accelerations in a quantitative manner, and attempts to identify numerical relationships between the hub accelerations and the fuselage vibrations. Also, this study was undertaken to obtain a better understanding of the basic dynamics underlying the main rotor-dependent fuselage vibration and the associated hub accelerations. The present study builds up on previous neural network studies that were conducted at NASA Ames in the areas of rotorcraft performance, acoustics, and dynamics (Refs. 2 to 8).

Flight test data from the NASA Army UH-60A Airloads Program (Refs. 9 and 10) were used in this study.

Reference 4 studied the neural network based modeling of the UH-60A peak, 4P pilot floor verical vibration (PVV) for real-time applications. In Ref. 4, the peak value of the pilot floor vertical vibration was used so as to better represent time varying maneuvers, such as a pull-up maneuver. Compared to Ref. 4 , the additional 
considerations present in this study are the effects due to the hub accelerations.

In this study, for purposes of modeling the UH-60A PVV and the hub accelerations, two databases were created. The first database included only level flight data. The second database included data from the entire flight envelope, including maneuver (or unsteady) conditions.

The present use of neural networks was justified because neural networks can perform multi-dimensional, nonlinear curve fitting. This feature is useful in the present study that seeks to identify smoothly varying relationships. The present work is considered to be a generic methodology and is not specific to the presently considered UH-60A configuration.

\section{Objectives}

The present neural network based representation (or modeling) study involving the helicopter N/rev peak, pilot floor vertical vibration (PVV) and the hub accelerations had the following four objectives:

1. Create a neural network training database for the hub accelerations. The corresponding training database for the PVV was created in Ref. 4.

2. Conduct exploratory studies involving the PVV and the advance ratio and the aircraft gross weight. Two databasés would be used, one for level flights and the other for "all flights," including maneuvers. For objective 2, only the PVV data contain maneuver effects, since neither the advance ratio nor the aircraft gross weight account for maneuver conditions.

3. Assess the data quality of the hub accelerations and obtain their neural network based representations.

4. Using the hub accelerations and flight condition parameters such as the advance ratio and gross weight, determine whether reasonably accurate analytical representations of the PVV could be obtained.

\section{Hub Acceleration and Pilot Vertical Vibration Databases}

The source of the hub accelerometer data was the NASA/Army UH-60A Airloads Program tlight test database (Refs. 9 and 10). The creation of the corresponding PVV database has been described in Ref. 4. The following flight conditions were included: level flights, rolls, pushovers, pull-ups, autorotations, and landing flares. These conditions approximate the entire UH-60A flight envelope.
The UH-60A hub accelerometers were mounted on a triaxial block glued to the main rotor shaft 4.5 inches from the center of rotation (Ref. 1). Three accelerometers (radial, tangential, and vertical) were used. Following Ref. 1, the tangential accelerometer measurements were used to present the in-plane response because it has a smaller centrifugal acceleration value than the radial sensor.

The present study thus considered the $3 \mathrm{P}$ and $5 \mathrm{P}$ tangential hub accelerations and the $4 \mathrm{P}$ vertical hub acceleration. The appropriate hub acceleration values were taken as those corresponding to the peak PVV (Ref. 4). Let the peak PVV occur at a time $t=\tau$. The appropriate hub acceleration was defined as that also occurring at time $t=\tau$. In general, the peak-PVVtime, $\tau$ was different for different maneuvers, and had to be individually determined.

\section{Maneuver Effect Factor}

The MEF, a non-dimensional parameter, was used to characterize helicopter maneuvers involving simultaneous non-zero roll-angle and pitch-rate. The MEF was used as an input in the MEF-related neural network. In the present study, the MEF was defined by the following equation (Ref. 4 ):

$$
\begin{aligned}
& \text { Maneuver effect factor, MEF }= \\
& \qquad \begin{array}{l}
{[1 / \cos (\text { roll-angle })] *} \\
{[1+(\text { pitch-rate } * \text { airspeed } / g)]}
\end{array}
\end{aligned}
$$

where " $\mathrm{g}$ " is the acceleration due to gravity. The purpose of the MEF was to compactly represent complex maneuvers using a single, physics-based parameter. Depending on the reference axes system used, other parameters can be derived, and this would result in slightly different formulations.

The number of the neural network training data points in the present study was over 200 . These points represent the entire training database. Approximately $25 \%$ of the training database involved maneuver related points. Here, maneuver related refers to a flight condition for which the maneuver effect factor MEF was not equal to 1 .

\section{Basic Variations: Hub Accelerations and Pilot Vertical Vibration}

Figures 1-4 show the variations of the flight test hub accelerations and the PVV versus the advance ratio. The data shown in Figs. $1-4$ use the 200 point flight database. Thus, in addition to the variation in the advance ratio covered in the figures, overall, these data 
involved variations in the gross weight, the RPM, the density ratio, the MEF, and the ascent/descent rate (and variations in the three control stick positions).

Figure 1 shows the $3 \mathrm{P}$ tangential hub acceleration variation with the advance ratio. These data were obtained with the 3P bifilars installed on the UH-60A (Ref. 9). In Fig. 1, the low speed "hump" due to rotor wake effects can be seen around an advance ratio of 0.09 (approximately $40 \mathrm{knots}$ ). Figure 2 shows the 5P tangential hub acceleration variation with the advance ratio. Compared to the 3P hub acceleration data in Fig. 1 , the SP hub acceleration data infFig. 2 appear to contain more scatter. This could due to the fact that the 3P bifilars bring in a forced response behavior that tends to smooth "out the $3 \mathrm{P}$ accelerations in the UH-60A Airloads Program the UH-60A@ nov hP bifilars installed. Figure 3 shows the 4 vertical hub acceleration variation with the advance ratio. Figure 4 shows the peak, 4P pilot floor vertical vibration, PVV, versus the advance ratio (Ref. 4).

\section{Neural Network Approach}

To accurately capture the required functional dependencies, the neural network inputs must be carefully selected and account for all important physical traits that are specific to the application. The important attributes of a neural network are its type (radial-basis function network or back-propagation network, etc.) and its complexity (i.e., the number of processing elements (PEs) and the number of hidden layers). The present overall neural network modeling approach (Refs. 2-8) consists of first determining the best type of neural network to be used and then simplifying the network as much as is practical.

Determining the best type of neural network usually involves selecting either a radial-basis function (RBF) or a back-propagation network. In the present study, the back-propagation type of network was used.

Simplifying the network involves reducing the number of PEs and in a few cases, the number of hidden layers. The number of PEs required depends on the specific application. The determination of the appropriate number of PEs is done by starting with a minimum number of PEs. Additional PEs are added to improve neural network performance by reducing the RMS error between the test data and the neural network predictions. Typically, five PEs are added at each step in this process. Adding two or three PEs at a time fine-tures the neural network.

If the correlation plot, comparing measured and predicted values, shows only small deviations from the 45-deg reference line, the neural network has produced an acceptable representation of the subject test data. If the plot shows points well off of the 45-deg line, poor quality test data may exist in the database. A detailed examination of the subject test database is then required to identify the source(s) of the errors associated with these test data.

For notation used in this paper, a neural network architecture such as "4-25-5-1" refers to a neural network with four inputs, twenty five processing elements (PEs) in the first hidden layer, five PEs in the second hidden layer, and one output.

The application of neural networks to full-scale helicopter flight test vibration and hub accelerations data was conducted using the neural networks package NeuralWorks Pro II/PLUS (version 5.2) by NeuralWare (Ref. 11). The present neural network RMS error was dimensionless and based on the squares of the errors for each processing element (PE) in the output layer. Generally, the RMS error was characterized by a monotonic decrease with the number of training iterations (Ref. 7). Also, any large differences in the magnitudes of the neural network variables were mitigated by appropriate scaling. In the present application, the cost function used in minimizing the RMS error had equally weighted individual contributions.

Three basic studies, taken up in order of increasing complexity, were conducted, and are described as follows:

i). An initial exploratory study was conducted to determine the relationships between the PVV and the advance ratio and the gross weight. Two sets of results, one for level flight and the other involving the entire database that included maneuver effects were obtained.

ii). A hub accelerations representation study was conducted using the three neural network input sets created in Ref. 4. These three input sets are listed later (Hub Accelerations Representation). The entire database was used. Results pertaining to only one of the input sets, namely, the MEF-input set, are presented in this paper.

iii). A study was conducted on using the hub accelerations along with the advance ratio and the gross weight to model the PVV. The entire database was used. The results from this third and final study would help determine whether the hub accelerations could be used to obtain the PVV.

\section{Results}

Bilot Vertical Vibration Exploratory Study 
This exploratory study had two parts. The first part involved level flight conditions and the second part involved the entire database including maneuver conditions. In this exploratory study, the PVV was the single neural network output.

\section{Level Elight}

The first part of this exploratory study involved level flight conditions, with varying gross weight and a constant RPM. Approximately 80 points were involved. Figures 5-7 show the results for these level flight cases.

Figure 5 shows the correlation plot from a SISO 1-10 5-1 back-propagation neural network in which the advance ratio was the single input. The backpropagation network was trained for 5 million iterations with a final RMS error of 0.17 . There does not appear to be a unique relationship between the advance ratio and the PVV.

Figure 6 shows the correlation plot from a SISO 1-105-1 back-propagation neural network in which the gross weight was the single input. The back-propagation network was trained for 5 million iterations with a final RMS ertor of 0.15. Again, not surprisingly, there does not appear to be a unique relationship between the gross weight and the PVV.

Figure 7 shows the correlation plot from a MISO 2-105.1 back-propagation neural network in which the advance ratio and the gross weight were the two inputs. The back-propagation network was trained for 1 million iterations with a final RMS error of 0.10 . Figure 7 shows that the advance ratio and the gross weight could represent the PVV for level flight conditions. The trained neural network, Fig. 7, can typically predict the PVV to within $+/-0.05 \mathrm{~g}$ 's, knowing only the advance ratio and the gross weight.

\section{All-Flights (Entire Database)}

The second part of this exploratory study involved all flight conditions considered in Ref. 4 . The entire database was used (200 points, including maneuvers). Figures $8-10$ show the corresponding results.

Figure 8 shows the correlation plot from a SISO 1-105-1 back-propagation neural network in which the advance ratio was the single input. The backpropagation network was trained for 5 million iterations with a final RMS error of 0.24 . There does not appear to be a unique relationship between the advance ratio and the PVV.

Figure 9 shows the correlation plot from a SISO 1-105-1 back-propagation neural network in which the gross weight was the single input. The back-propagation network was trained for 5 million iterations with a final RMS error of 0.25 . There does not appear to be a unique relationship between the gross weight and the PVV.

Figure 10 shows the correlation plot from a MISO 2. 10-5-1 back-propagation neural network in which the advance ratio and the gross weight were the two inputs. The back-propagation network was trained for 5 million iterations with a final RMS enror of 0.11 . Figure 10 shows that the advance ratio and the gross weight can reasonably predict the PVV for the entire flight database, maneuvers included. Compared to the entire database correlation shown in Fig. 10, the level flight correlation shown in Fig. 7 is "cleaner." The good correlation seen in Fig. 10 is encouraging, even though the neural network inputs (advance ratio and gross weight) do not account for maneuver effects.

For completeness, an existing correlation result based on a more complex physical model that accounts for maneuver effects (and other effects noted below) is shown in Fig. 11, taken from Ref. 4. Figure 11 shows the correlation plot from a MISO 6-10-5-1 backpropagation neural network. The back-propagation network was trained for 4 million iterations with a final RMS"error of 0.07 . The six inputs were as follows: the advance ratio, the gross weight, the RPM, density ratio, the MEF, and the ascent/descent rate. In Ref. 4, it was concluded that the Fig. 11 representation using the MEF was the best representation in terms of modeling an unsteady maneuver such as a pull-up. Figure 12, also from Ref. 4, shows the successful, quasi-static modeling of the unsteady PVV for a pull-up maneuver at 120 knots. Such fidelity in predicting the pilot floor vertical vibrations shows considerable promise in using neural networks to obtain the UH-60A fuselage vibrations.

\section{Hub Accelerations Representation}

The quality of the hub accelerations flight test data was assessed and their numerical representations were obtained. Three neural network databases were created to obtain the present three representations of the hub accelerations. The entire database was used (200 points, including maneuvers). The three input lists are given as follows:

Input List 1: The advance ratio, the RPM, the density ratio, the three pilot control stick positions, and the ascentdescent rate ( 7 inputs).

Input List 2: The Input List 1 above, with the gross weight additionally included ( 8 inputs). 
Input List 3: The Input List 2 above with the three pilot control stick positions removed, and with the maneuver effect factor, MEF, additionally included ( 6 inputs).

Results were obtained using all three input sets. This paper contains results using the third input set above since this gave the best results for unsteady maneuver conditions. Figures 13-15 show the correlation plots presently obtained using the MEF approach. The correlation plots in Figs. 13-15 were obtained from a MIMO 6-25-20-3 back-propagation neural network. The three neural network outputs were as follows: the $3 \mathrm{P}$ and $5 \mathrm{P}$ tangential hub accelerations and the $4 \mathrm{P}$ vertical hub acceleration. The back-propagation network was trained for 3 million iterations with a final RMS error of 0.06 . The figures show that the errorband for the hub accelerations was $+/-0.05 \mathrm{~g}$ 's.

Overall, the hub acceleration flight test "data quality" was assessed as being very acceptable (Figs. 13-15). There were no identifiable poor quality data points such as that discussed earlier (Neural Network Approach). As noted in Ref. 2 , the analyst should not solely rely on the neural network based correlation to eliminate poor quality test data. The present process does, however, contribute to data assessment. Finally, the Fig. 13-15 results imply that for the UH-60A, numerical relationships (the identification model) relating the hub accelerations to the flight condition parameters have been obtained.

\section{Relationships Between Hub Accelerations and Pilot Vertical Vibration}

The objective was to represent the PVV using the $3 P$ and $5 \mathrm{P}$ tangential hub accelerations and the $4 \mathrm{P}$ vertical hub acceleration as the three "core" inputs. Four cases were created, with their inputs listed as follows:

Case 1 inputs: the three hub accelerations ( 3 inputs).

Case 2 inputs: the three hub accelerations and the advance ratio (4 inputs).

Case 3 inputs: the three hub accelerations and the gross weight ( 4 inputs).

Case 4 inputs: the three hub accelerations, the advance ratio and the gross weight (5 inputs).

The PVV was the single neural network output, and the entire database was used (200 points, including maneuvers)

Figures $16-19$ show the correlation plots obtained using the four input sets given above. Figure 16 shows the correlation plot from a MISO 3-10-5-1 back- propagation neural network with the case 1 inputs (the three hub accelerations). This case was difficult to train (the training error was always high) and consequently, the number of iterations was varied to determine the network with the smallest error. Figure 16 shows the results with the back-propagation network trained for 3 million iterations with a final RMS error of 0.18 , the smallest error obtainable. For this case, increasing the number of iterations to 5 million (and subsequently, to 6 million) resulted in a slightly larger RMS error, 0.20 . Only the 3-million iteration results are reported in this paper. Figure 16 shows that there does not appear to exist a unique relationships between the hub accelerations and the PVV. As such, it can be suggested that the hub accelerations contain some basic information that depends on the flight condition.

Figure 17 shows the correlation plot from a MISO 410-5-1 back-propagation neural network with the case 2 inputs (the three hub accelerations and the advance ratio). The back-propagation network was trained for 5 million iterations with a final RMS error of 0.11 . Figure 17 shows that the hub accelerations and the advance ratio could represent the PVV. The Fig. 17 correlation is very encouraging because it appears that the physics of the all-airspeeds-behavior of the PVV is being captured by the advance ratio (in combination with the hub accelerations).

Figure 18 shows the correlation plot from a MISO 410-5-1 back-propagation neural network with the case 3 inputs (the three hub accelerations and the gross weight). The back-propagation network was trained for 5 million iterations with a final RMS error of 0.10 . Figure 18 shows that the hub accelerations and the gross weight could represent the PVV. Unlike the advance ratio used in obtaining the Fig. 17 results, the gross weight used in obtaining the Fig. 18 results has very little or no physical significance. Thus, the gross weight may have a secondary meaning as a unique "identifier" that helps in neural network training. The hub accelerations themselves are likely to contain basic information that when combined with an appropriate identifier can produce an acceptable correlation.

Figure 19 shows the correlation plot from a MISO 5 10-6-1 back-propagation neural network with the case 4 inputs (the three hub accelerations along with the advance ratio and the gross weight). The backpropagation network was trained for 5 million iterations with a final RMS error of 0.08 . Figure 19 shows that the hub accelerations along with the advance ratio, and the gross weight could represent the PVV. Compared to the Fig. 17 correlation (involving the hub accelerations and the advance ratio), the Fig. 19 correlation is not surprising. This is because the hub accelerations are likely to contain a substantial amount of basic information and only a minimum amount of 
additional information (such as the advance ratio, etc.) is required to produce neural network based representations. Also, the correlation shown in Fig. 11 uses the maneuver effect factor MEF whereas the correlation shown in Fig. 19 uses the hub accelerations (along with the advance ratio and the gross weight). Both correlation results were obtained such that they fell within $\mathrm{a}+/-0.05 \mathrm{~g}$ 's error band and thus are comparable to each other. Hence, it can be suggested that the hub accelerations contain maneuver effects information reflecting load factor effects.

Selected results are shown in Table 1 in numerical form to show typical neural network predictions for constant flight conditions. The test PVV's for four flight conditions and the corresponding neural network based PVV's are shown in Table 1. The neural network models for which the predictions were obtained are noted in Table 1. These models are as follows: the advance ratio and gross weight model (Fig. 10), the Ref. $4 \mathrm{MEF}$ model (present Fig. 11), and the hub accelerations along with advance ratio and the gross weight model (Fig. 19). The present neural network based models were accurate to within $+/-0.03 \mathrm{~g}$ 's for high-speed level flight, descent, climb, and a constant turn flight condition, Table 1. The Ref. 4 model that used the MEF and the present model that used the hub accelerations along with advance ratio and the gross weight gave the best PVV predictions.

\section{Conclusions}

Full-scale, flight test based peak, 4P pilot floor vertical vibration (PVV) and the corresponding hub accelerations were considered in this study for modeling purposes. The quality of the hub accelerations data was assessed. The present physics-based, quantitative effort represents the first systematic study involving hub accelerations. The flight conditions considered in the present study were as follows: level flights, rolls, pushovers, pullups, autorotations, and landing flares. A step-by-step approach in constructing the individual "example" or "test" cases was followed in this study. Basically, three "test" cases were considered.

In the first case, the PVV was considered by itself for modeling. Level flight conditions and those including maneuvers were separately considered. It was found that neither the advance ratio nor the gross weight alone could be used to represent the PVV. However, the advance ratio and the gross weight could be used to represent the PVV of virtually the entire UH-60A Airloads Program database.

In the second case, the three hub accelerations were considered and it was determined that the quality of the hub accelerations data was very acceptable. The hub accelerations data were successfully modeled. Here, the three input lists used in modeling the hub accelerations were the same as those associated with the three databases created in Ref. 4. Three sets of hub accelerations representation results were thus obtained. The present paper contains results obtained using one of the three input lists that was shown in Ref. 4 to give the best results for a maneuver or unsteady flight condition (specifically, a pull up). Thus, the hub accelerations results reported in this paper were based on the above maneuver effect factor input list: the advance ratio, the gross weight, the RPM, the density ratio, the $M E F$, and the ascent/descent rate.

In the third and final case, the relationships between the hub accelerations and the PVV were considered. It was found that the hub accelerations alone could not represent the PVV. Thus, the hub accelerations alone do not drive the PVV. The hub accelerations, along with either the advance ratio or the gross weight or both, could be used to represent the PVV. The hub accelerations are clearly a factor in determining the PVV.

It appears from the present study that the hub accelerations are likely to contain a substantial amount of important information that can be used to model helicopter vibration. For example, the hub accelerations may contain maneuver effects information reflecting load factor effects.

\section{References}

1. Studebaker, K., "A Survey of Hub Vibration for the UH-60A Airloads Research Aircraft, American Helicopter Society International Aeromechanics Specialists Conference, San Francisco, Califomia, January 1994.

2. Kottapalli, S. “Neural Network Research on Validating Experimental Tilt-Rotor Performance," Joumal of the American Helicopter Society, Vol. 45 , No. 3, July 2000 , pp. 199-206. AIAA $16^{\text {th }}$ Applied Aerodynamics Conference, AIAA-98-2418, Albuquerque, New Mexico, June 1998.

3. Kottapalli, S. and Kitaplioglu, C., "Neural Network Representation of Experimental Tilt-Rotor Noise," $6^{\text {th }}$ ALAA/CEAS Aeroacoustics Conference, AIAA-2000-1924, Maui, Hawaii, June 2000.

4. Kottapalli, S., "Neural-Network-Based Modeling of Rotorcraft Vibration for Real-Time Applications," ALAA Modeling and Simulation Technologies Conference, AIAA-2000-4305, Denver, Colorado, August 2000.

5. Kottapalli, S., "Exploratory Study on Neural Control of Rotor Noise and Hub Loads," American 
Helicopter Society Technical Specialists' Meeting for Rotorcraft Acoustics and Aerodynamics, Williamsburg, Virginia, October 1997.

6. Kottapalli, S., "Identification and Control of Rotorcraft Hub Loads Using Neural Networks," American Helicopter Society 53 ${ }^{\text {rd }}$ Annual Forum, Virginia Beach, Virginia, April-May 1997.

7. Kottapalli, S., Abrego, A., and Jacklin, S., "Application of Neural Networks to Model and Predict Rotorcraft Hub Loads," American Helicopter Society Second International Aeromechanics Specialists Conference, Bridgeport, Connecticut, October 1995.

8. Kottapalli, S, "Application of Neural Networks to Aeromechanics Problems," 24 $4^{\text {th }}$ European
Rotorcraft Forum, Marseilles, France, September 1998.

9. Kufeld, R.M., Balough, D.L., Cross, J.L., Studebaker, K.F., Jennison, C.D., and Bousman, W.G., "Flight Testing of the UH-60A Airloads Aircraft," American Helicopter Society $50^{\text {th }}$ Annual Forum, Washington, D.C., May 1994.

10. Bondi, M.J. and Bjorkman, W.S., "TRENDS, A Flight Test Relational Database," User's Guide and Reference Manual, NASA TM 108806, June 1994.

11. NeuralWorks Manuals: a) Reference Guide b) Neural Computing c) Using NeuralWorks, NeuralWare, Inc., Pittsburgh, Pennsylvania, 1995. 
Table 1. Neural Network Based Predictions of Pilot Floor Vertical Vibration, PVV, g's

\begin{tabular}{lcccc}
\hline \hline Flight Condition & Test & $\begin{array}{c}\text { Advance Ratio }+ \\
\text { Gross Weight } \\
\text { (Fig. 10) }\end{array}$ & $\begin{array}{c}\text { Maneuver-Effect- } \\
\text { Factor, Ref. 4 } \\
\text { (Fig. 11) }\end{array}$ & $\begin{array}{c}\text { Hub Accels. + Advance- } \\
\text { Ratio + Gross Weight } \\
\text { (Fig. 19) }\end{array}$ \\
\hline Level flight, 135 knots & 0.10 & 0.10 & 0.09 & 0.09 \\
Descent, 160 knots & 0.25 & 0.22 & 0.24 & 0.25 \\
Climb, 62 knots & 0.12 & 0.09 & 0.12 & 0.13 \\
Turn, 45 deg, 120 knots & 0.13 & 0.10 & 0.13 & 0.14 \\
\hline \hline
\end{tabular}




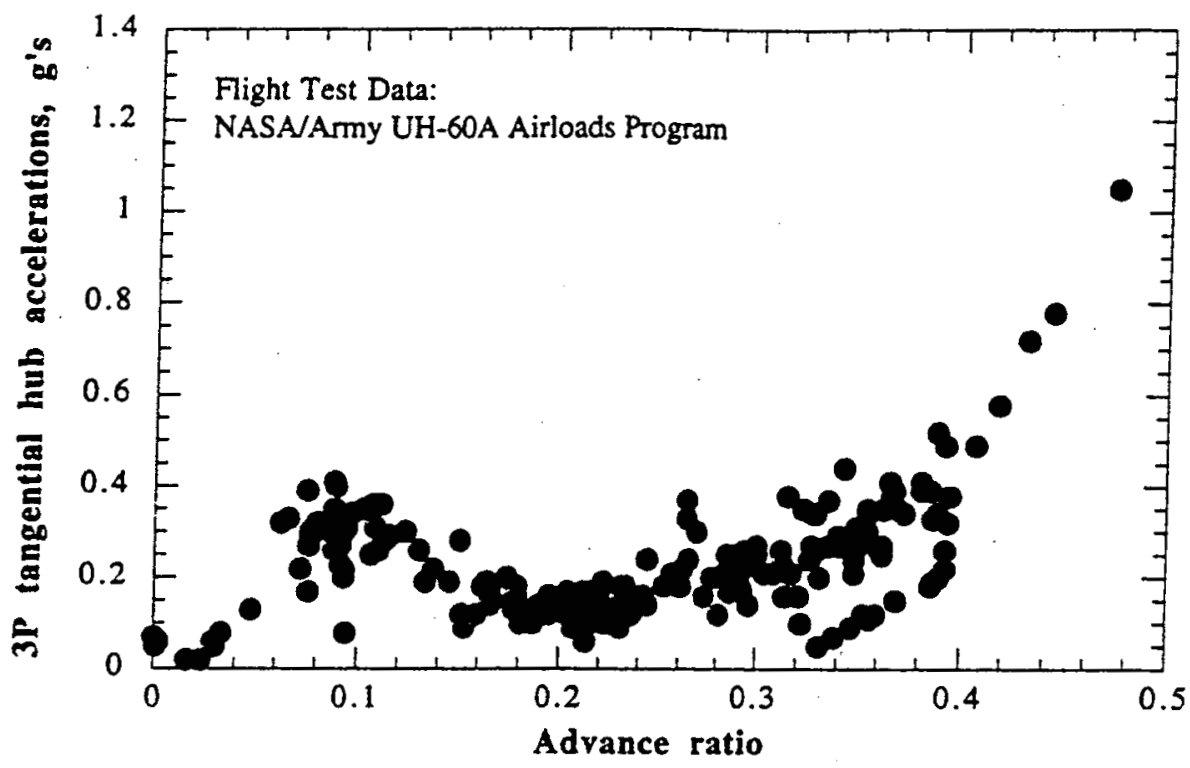

Fig. 1. UH-60A 3P tangential hub acceleration variation with advance ratio.

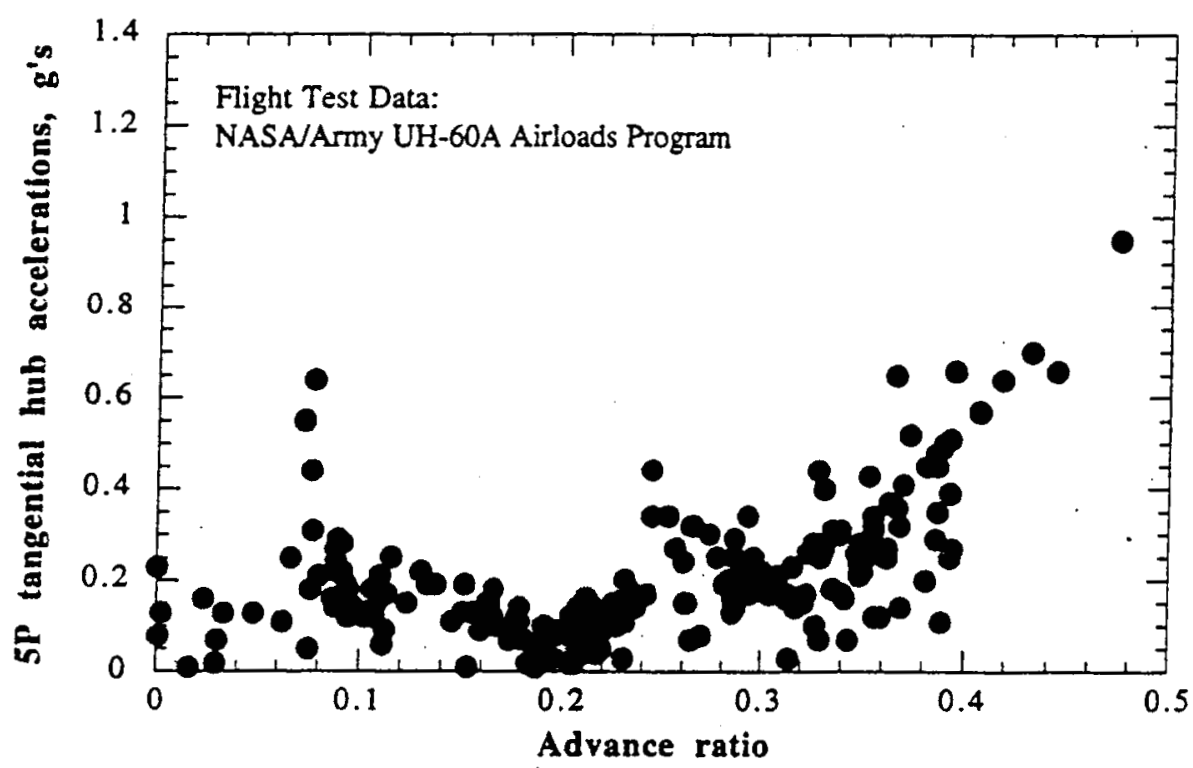

Fig. 2. UH-60A 5P tangential hub acceleration variation with advance ratio. 


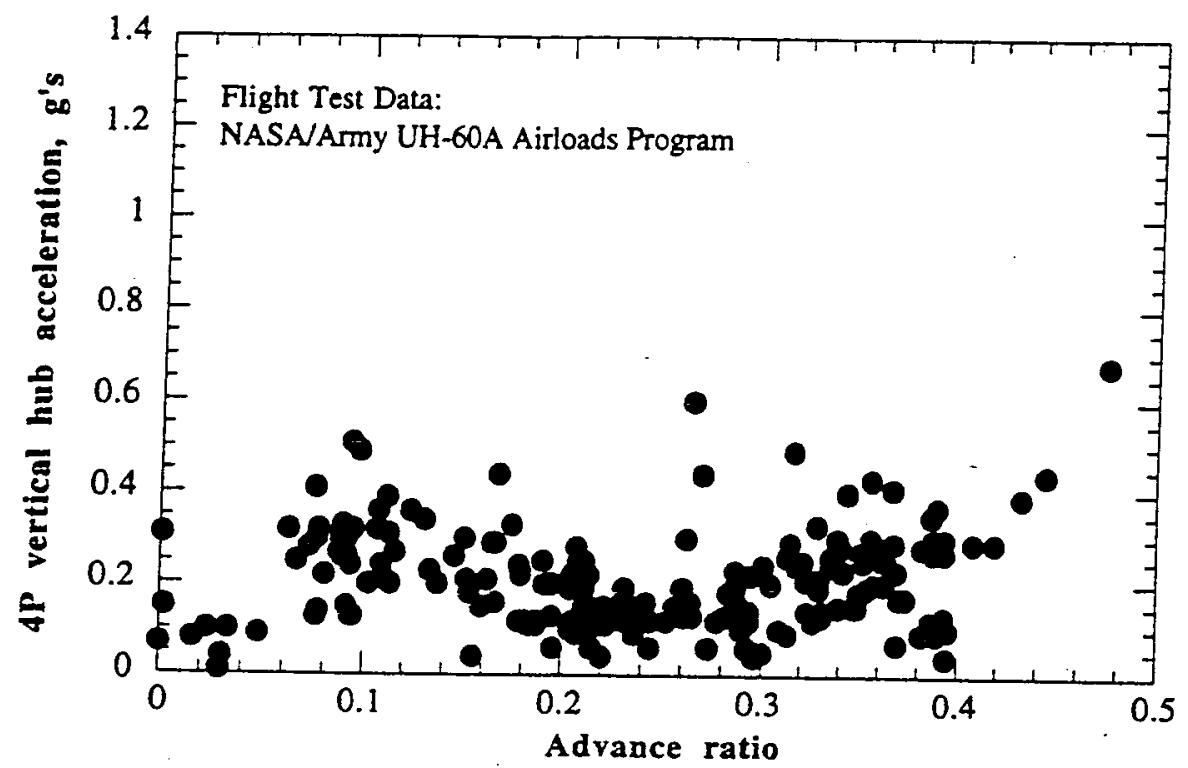

Fig. 3. UH-60A $4 \mathrm{P}$ vertical hub acceleration variation with advance ratio.

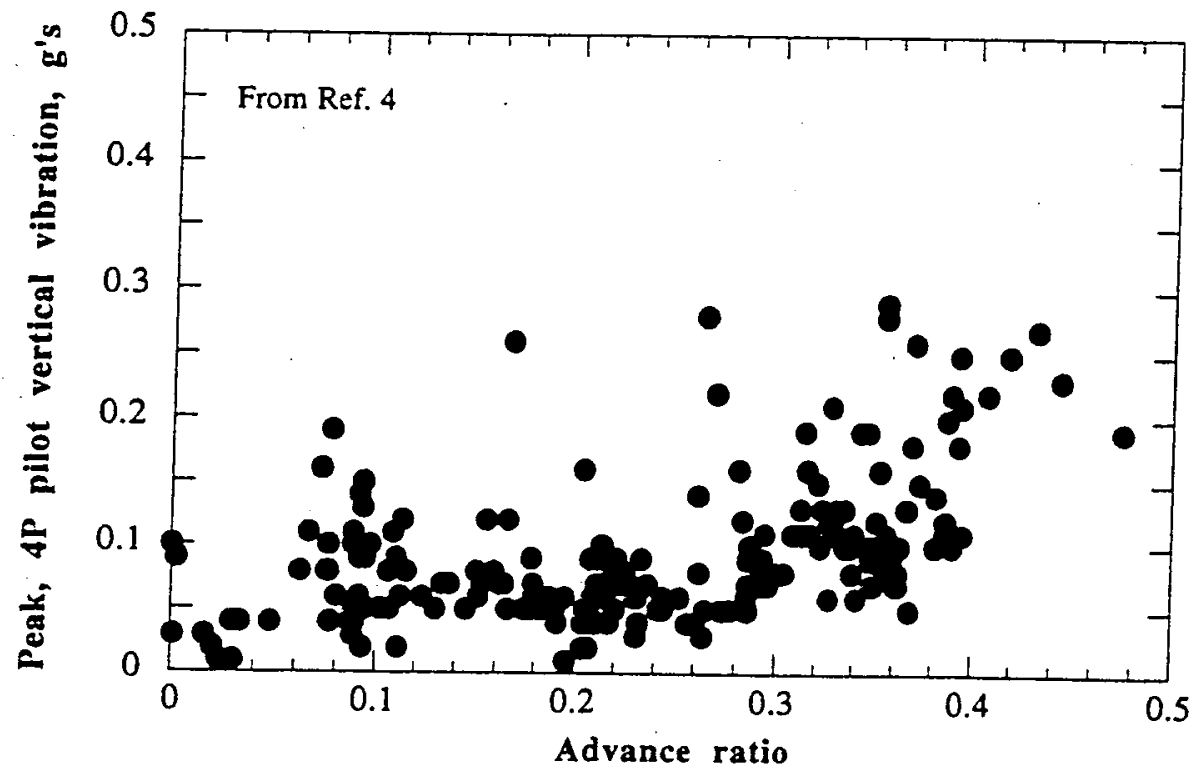

Fig. 4. UH-60A peak, 4P pilot floor vertical vibration, PVV, variation with advance ratio (Ref. 4). 


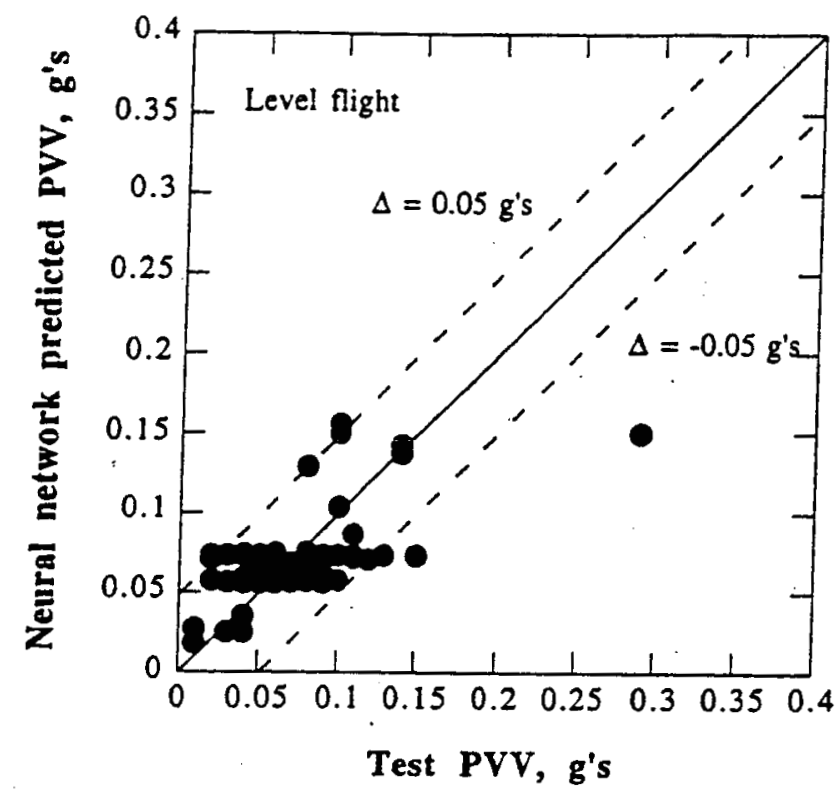

Fig. 5. PVV correlation using advance ratio, level fight.

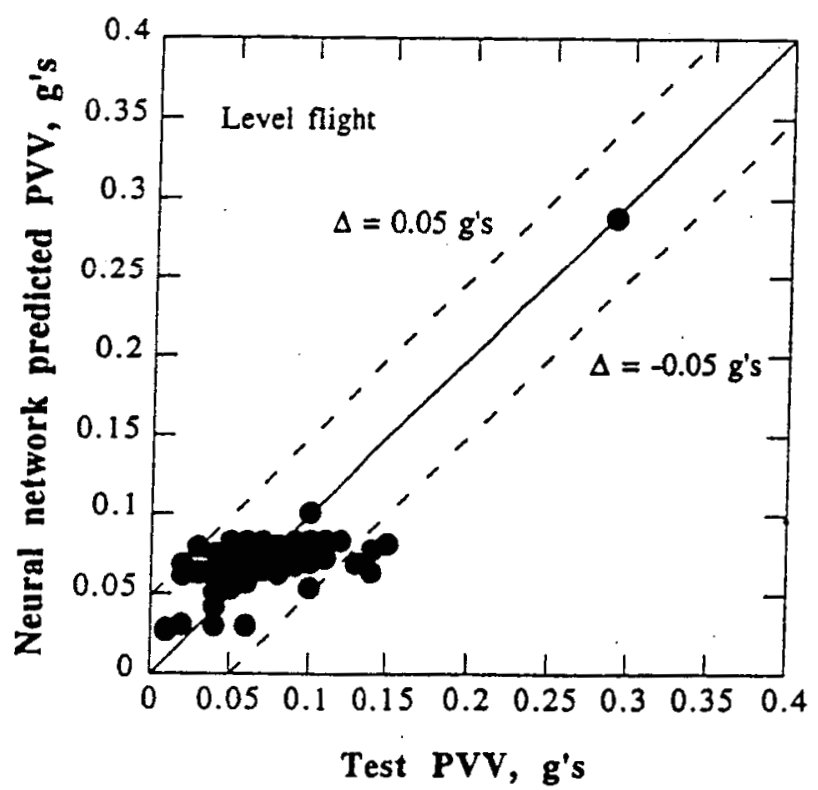

Fig. 6. PVV correlation using gross weight, level flight. 


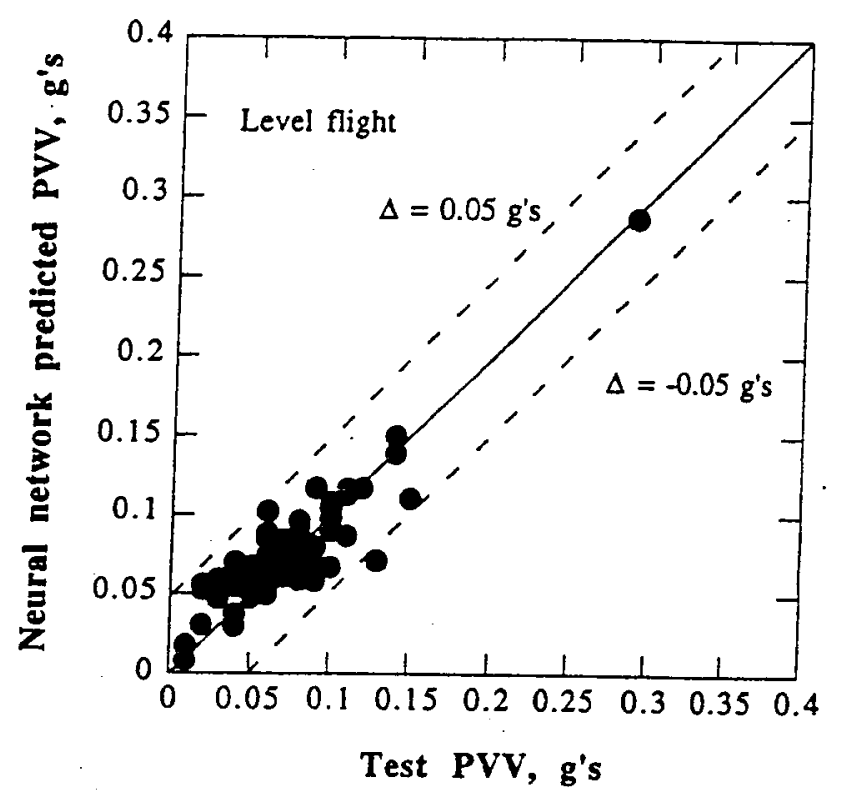

Fig. 7. PVV correlation using advance ratio and gross weight, level flight.

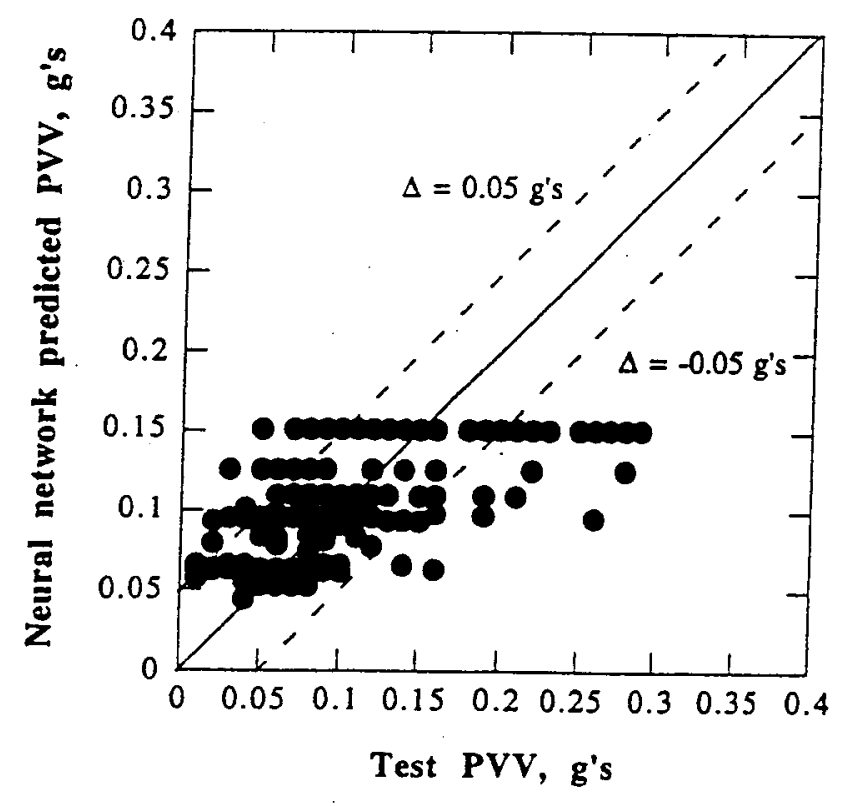

Fig. 8. PVV correlation using advance ratio. 


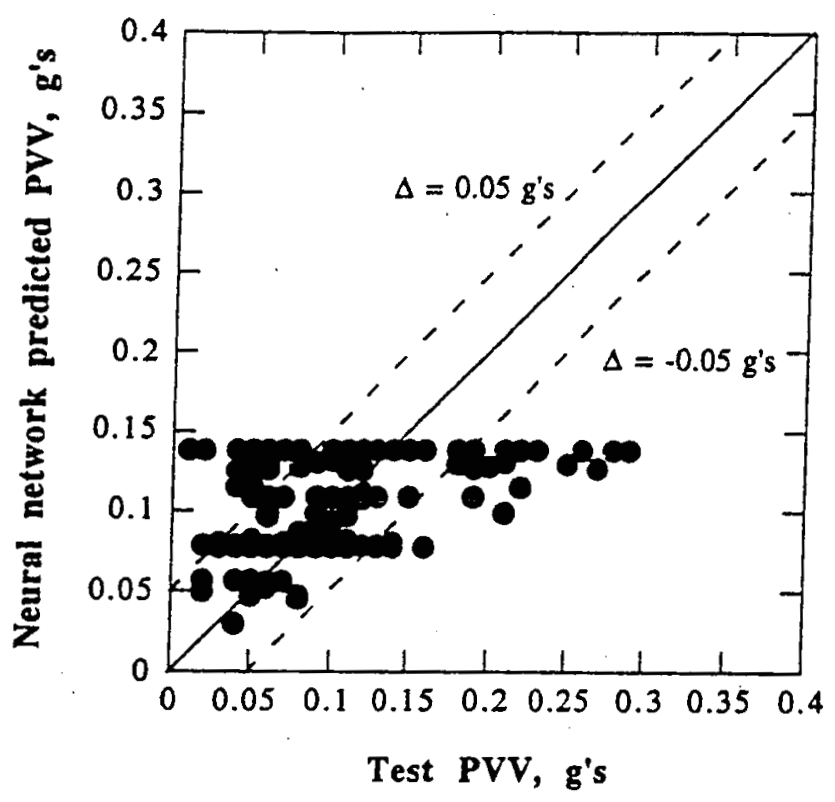

Fig. 9. PVV correlation using gross weight.

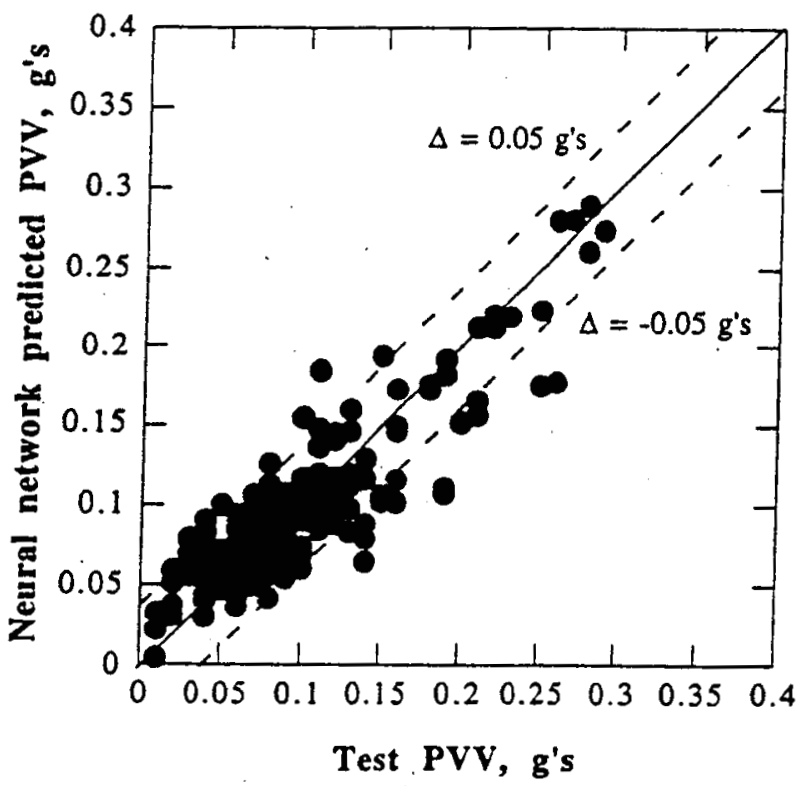

Fig. 10. PVV correlation using advance ratio and gross weight. 


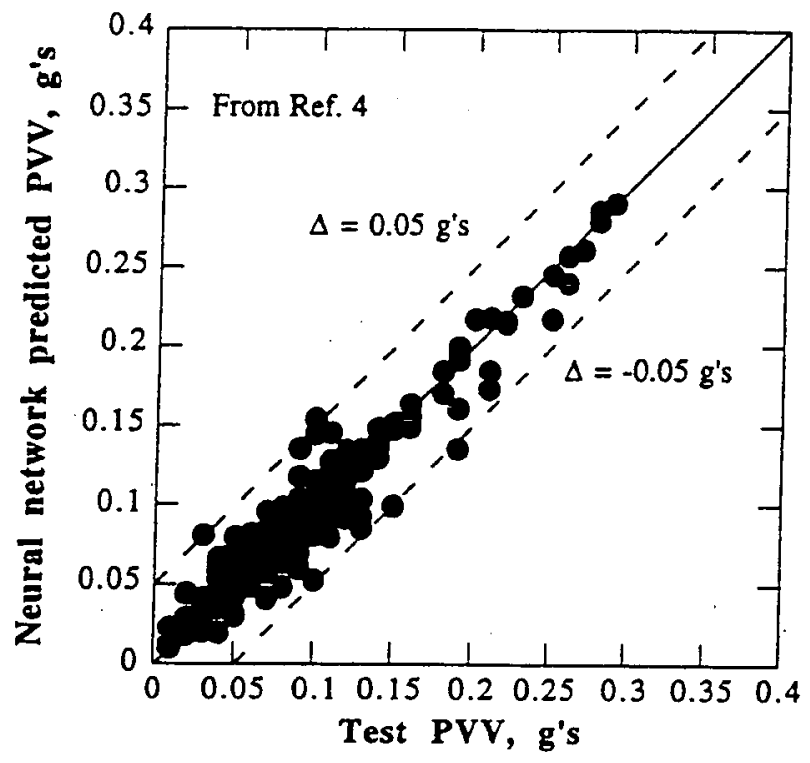

Fig. 11. PVV correlation using maneuver effect factor, MEF.

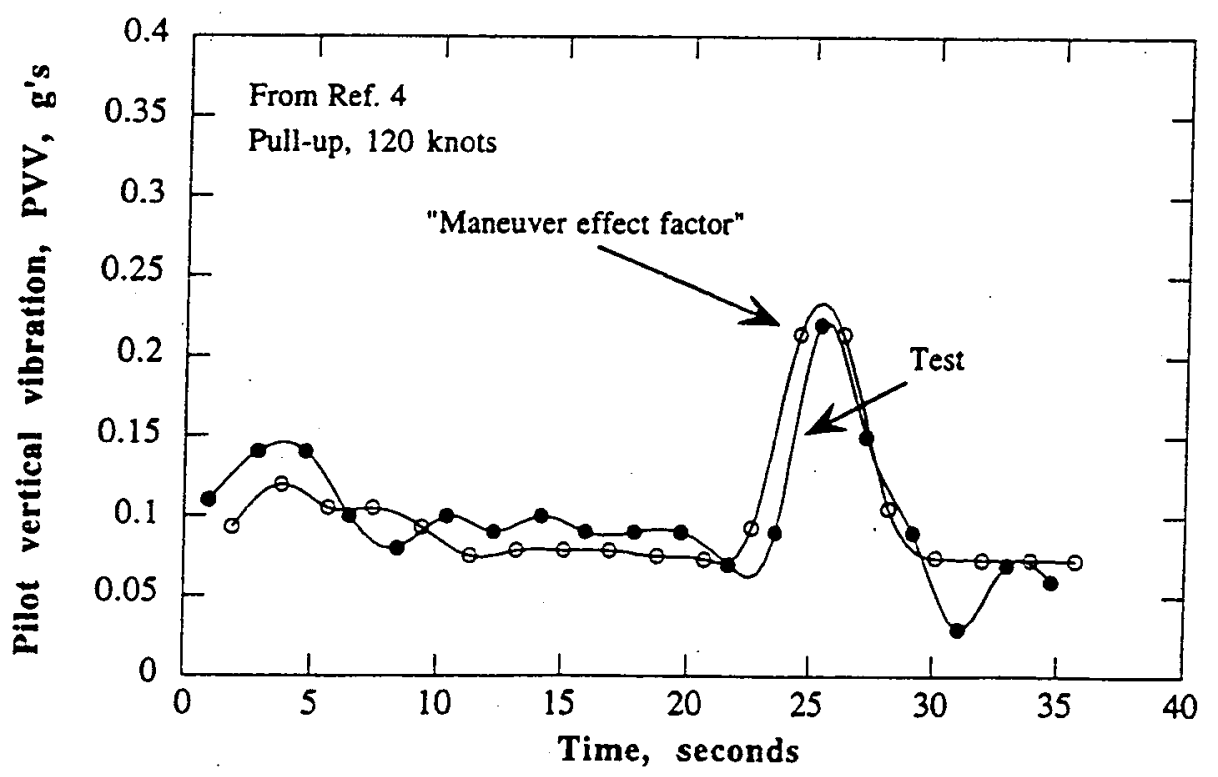

Fig. 12. Quasi-static prediction of PVV using MEF, unsteady pull-up (Ref. 4). 


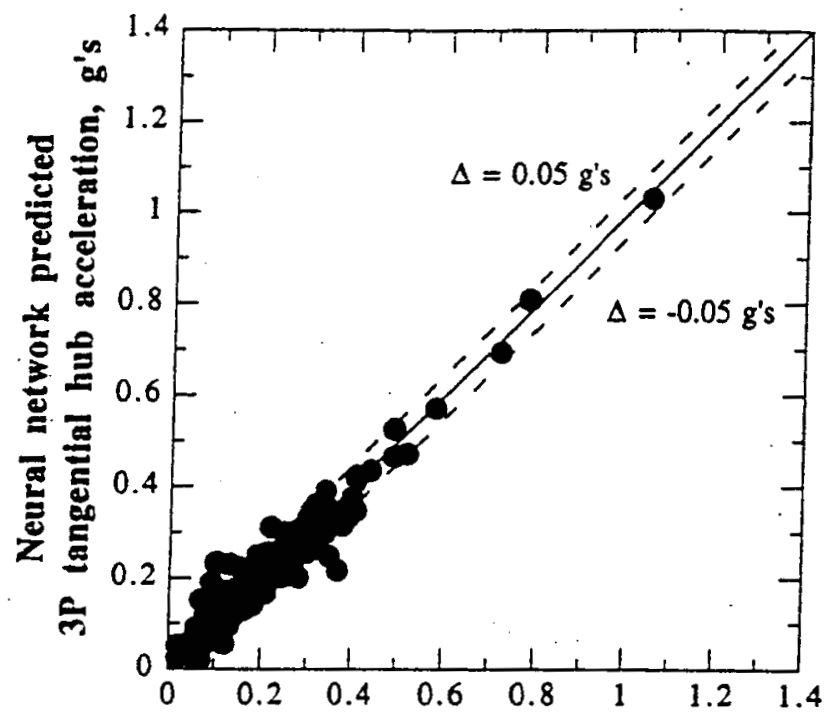

Test 3P tangential hub acceleration, g's

Fig. 13. 3P tangential hub acceleration correlation using maneuver effect factor.

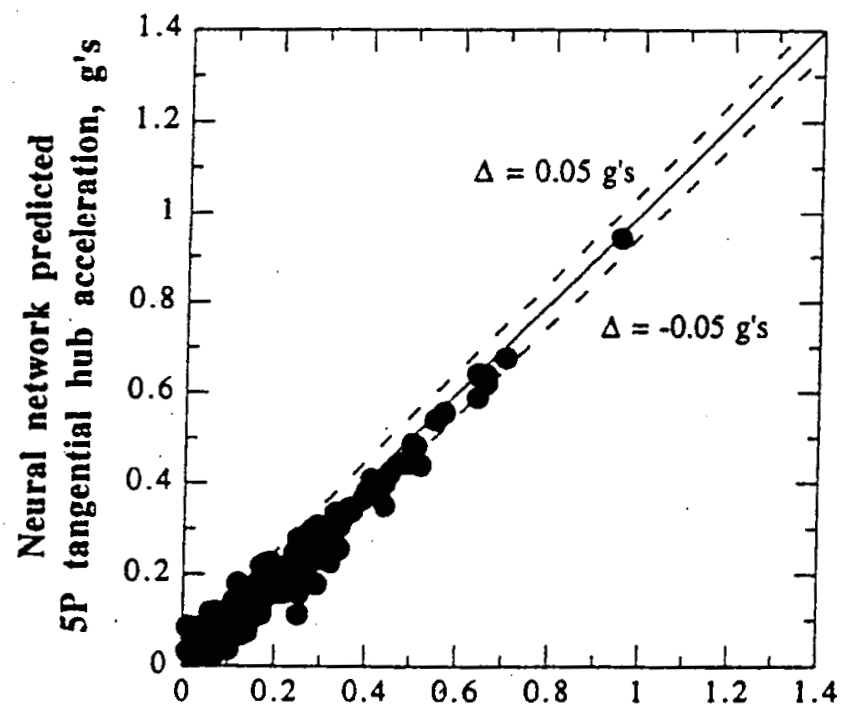

Test $5 \mathbf{P}$ tangential hub acceleration, $\mathbf{g}$ 's

Fig. 14. 5P tangential hub acceleration correlation using maneuver effect factor. 


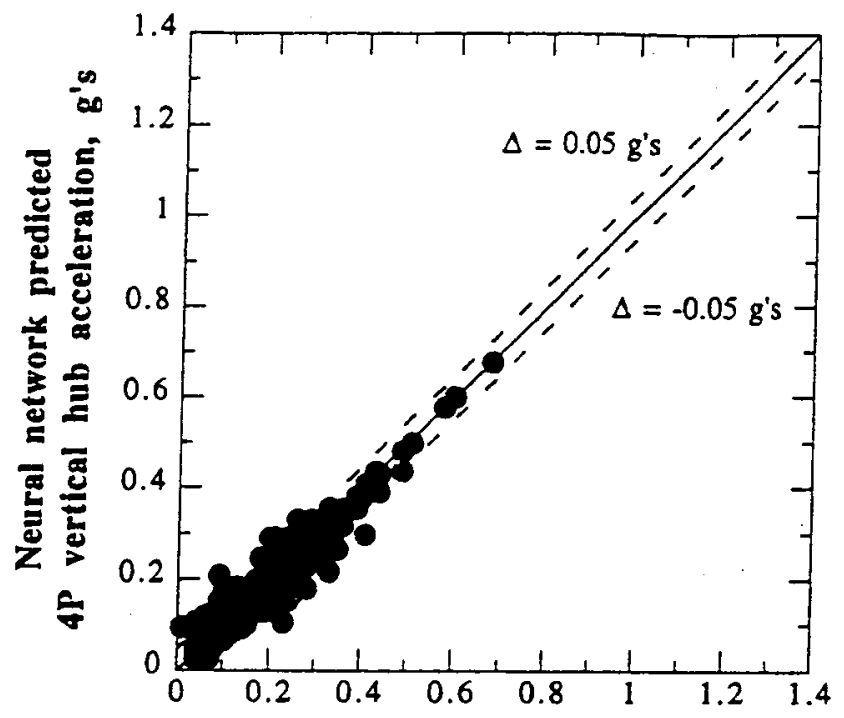

Test 4P vertical hub acceleration, g's

Fig. 15. 4P vertical hub acceleration correlation using maneuver effect factor.

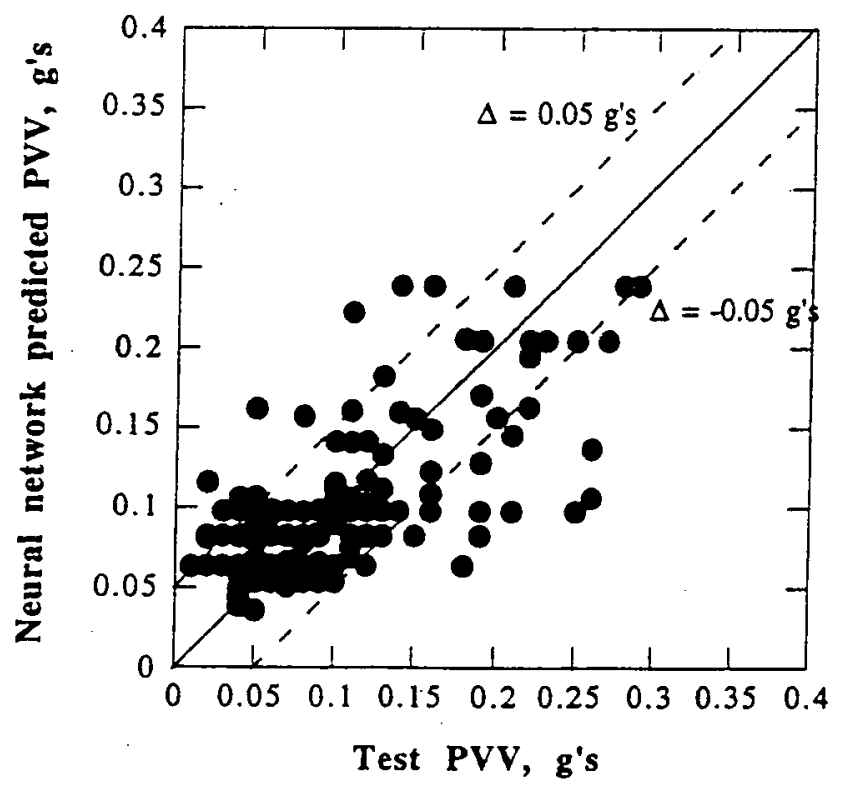

Fig. 16. PVV correlation using hub accelerations. 


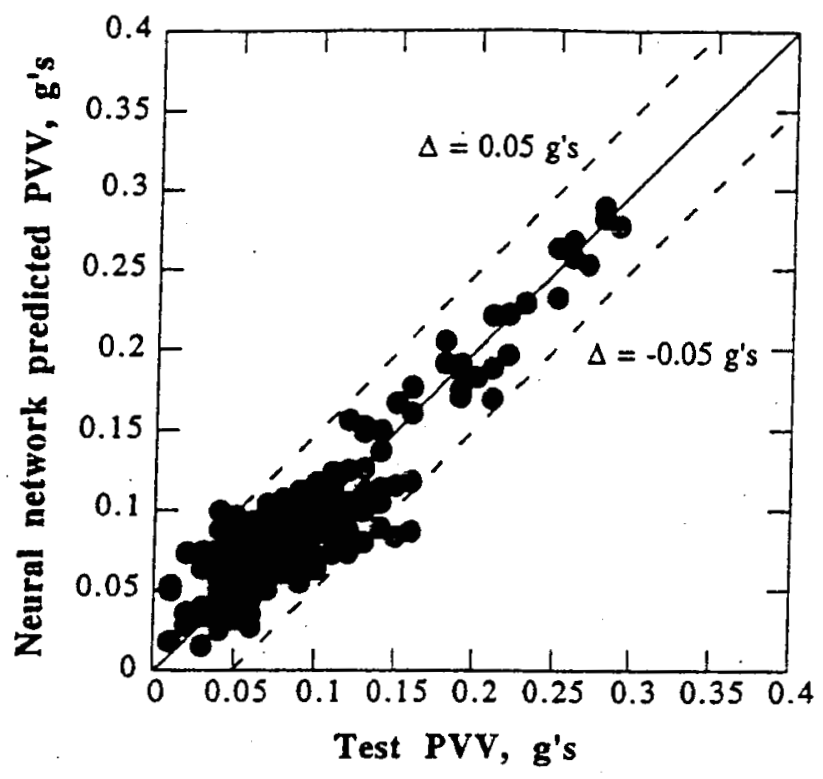

Fig. 17. PVV correlation using hub accelerations and advance ratio.

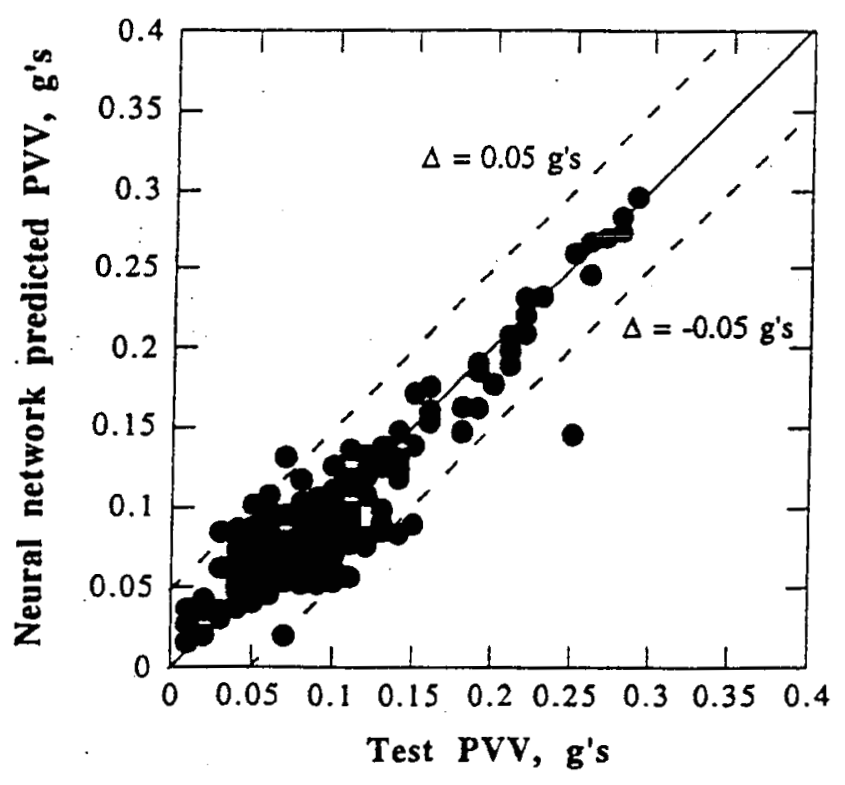

Fig. 18. PVV correlation using hub accelerations and gross weight. 


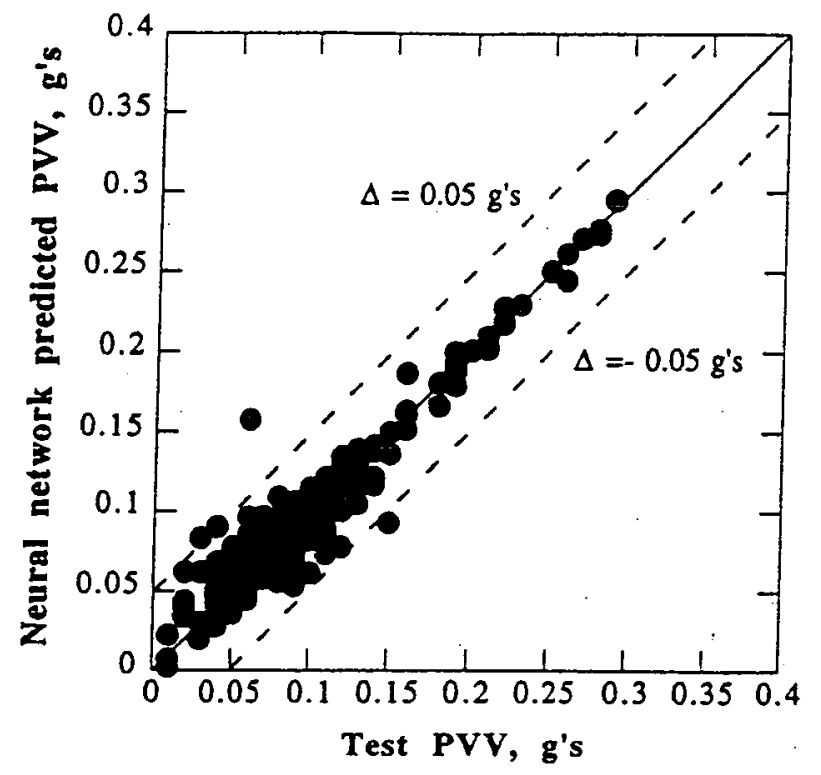

Fig. 19. PVV correlation using hub accelerations along with advance ratio and gross weight. 